\title{
The Need for Psycho-Oncological Support in Breast Cancer Patients - A Longitudinal Study in an Outpatient- Setting
}

\author{
Christian M. Kurbacher ${ }^{1 *}$, Ralf Reichelt ${ }^{2}$ and Roland Schnell ${ }^{3}$ \\ ${ }^{1}$ Gynäkologisches Zentrum Bonn-Friedensplatz, Bonn, Germany \\ 2 Onkotrakt AG, Hamburg, Germany \\ ${ }^{3}$ Praxis Internistischer Onkologie und Hämatologie (PIOH), Frechen, Germany
}

Corresponding Author: Christian M. Kurbacher, Gynäkologisches Zentrum Bonn-Friedensplatz, Bonn, Germany, E-mail: Praxis.Kurbacher@online.ms

Received Date: January 24, 2019; Accepted Date: February 12, 2019; Published Date: February 13, 2019.

Citation: Christian M. Kurbacher, Ralf Reichelt and Roland Schnell, The need for psycho-oncological support in breast cancer patients - a longitudinal study in an outpatient- setting. J. Psychology and Mental Health Care.Doi: 10.31579/ 2637-8892.19/012

Copyright: (c) 2019 Christian M. Kurbacher, This is an open-access article distributed under the terms of the Creative Commons Attribution License, which permits unrestricted use, distribution, and reproduction in any medium, provided the original author and source are credited.

\begin{abstract}
Objective Breast cancer is frequently associated with psycho-oncological burden for the affected patients. Severity and temporal dimension are often not readily assessable, as many patients do not express their need for professional counseling. Monitoring usually ends together with the patients' intensive medical cancer therapy. Thus, information on the longitudinal need for psycho-oncological support is limited. The aim of this questionnaire- based observational study was to identify breast cancer patients in need of psycho-oncological support and to monitor these patients in the long-term.

Methods A total of 94 patients treated in two oncological practices in Germany between May 2011 and July 2015 were included. 61 patients had primary breast cancer and 33 presented with recurrent/ metastatic disease. To assess the patients burden, the standardized self-rating Questionnaire on Stress in Cancer Patients - short form (QSC-R10) was to be answered by each patient at different time points.

Results Of $41 \%(n=39)$ initially burdened patients treated either in a curative or in a palliative setting, $67 \%(n=26)$ still required psycho-oncological support at the time of the second survey.

Conclusions Psycho-oncological burden is common in breast cancer patients and the need for support persists over a long period of time. In the future, this temporal dimension has to be adequately addressed after the completion of their intensive medical cancer therapy. Thus, the long-term monitoring of cancer patients with the QSC-R10 is strongly recommended.
\end{abstract}

Keywords: breast cancer; curative/ palliative treatment; psycho-oncological burden; psycho-oncological screening; QSC-R10

\section{Introduction}

In Germany, breast cancer is by far the most common cancer disease in women with about 70,000 new cases per year [1]. In a large proportion of the affected patients, the diagnosis is accompanied by fear of death, loss of control, isolation, hopelessness, and depression [2]. Secondary to cancer diagnosis and treatment, around $30 \%$ of all cancer patients suffer from significant psycho-social distress or require psycho-oncological support $[3,4,5]$. However, the identification of burdened patients is generally very difficult without implementing validated screening instruments, as many of the affected patients do not express their need for professional counselling and objective characteristics of the disease often do not correlate with the subjective perception and reactions of the patients[4].

Since the psychological condition has a major impact on the individual patient's quality of life and may well affect the course of the disease [6], it is of particular importance to detect psycho-oncological burden at an early stage and thus to ensure psychological support in all phases of the cancer experience. According to German national and international guidelines, screening for psycho-oncological burden is recommended for all breast cancer patients [7]. In this context, appropriate screening procedures should be used in addition to clinical observations [5]. However, as many patients are incapable or unwilling to verbalize their feelings and emotions to their physicians, family and medical care personal, self-assessment questionnaires may more realistically reflect the validated instruments such as the Hospital Anxiety and Depression Scale (HADS), the National Comprehensive Cancer Network Distress-Thermometer (NCCN DT), and the Questionnaire on Stress in Cancer Patients (QSC-R23) have been developed in the past [19] .

In the year 2008, the self-rating questionnaire QSC-R10 - which is the short form of the QSC- R23 - was developed [4]. Compared to the QSC-23, it is much shorter, which means less effort both for patients and evaluating persons[4].

Since its implementation, the QSC-R10 is highly accepted among professionals and patients within the distress-screening procedures in comprehensive cancer centers in Germany [4]. Due to the high acceptance and the minimal effort for answering the questionnaire, the QSC- R10 seems to be suitable for the long-term monitoring of cancer patients. However, to our knowledge, concise information on the longitudinal need for psychological intervention in breast cancer patients treated on an outpatient basis is rare. Therefore, the present prospective observational study was conducted to identify patients in need of psycho-oncological support at an early stage, and to monitor these patients over a longer period of time by using the QSC- R10. 
The present study was conducted from May 2011 until July 2015 in two German oncological practices, in order to assess the psycho-oncological burden of breast cancer patients. In this context, a total number of 94 adult breast cancer patients treated in a curative $(\mathrm{N}=61)$ or palliative intent $(\mathrm{N}=33)$ were asked to answer the self-assessment Questionnaire on Stress in Cancer Patients - short form (Table 1) at least twice throughout the observation period. The patients' burden was classified according to Herschbach \& Weis, 2010 [5]: patients were considered as burdened/in need of psycho-oncological support if $\geq 1$ item was rated with a score of 5, or 3 items were rated with a score of 4 . A multidimensional burden was diagnosed at a total score of $>14$. Throughout the observation period, patients could receive psycho- oncological support.

Prior to study entry, informed consent into the data collection had been obtained from each participating patient. This study was conducted according to the Declaration of Helsinki and in regard to both national and regional ethical guidelines. It was approved by the regional ethics committee (Ethics Committee of the North Rhine Medical Council, study number 2010310).

Here is a list of potentially stressful situations which might apply in your daily life. For each situ-
ation, please decide whether the situation applies to you. If so, please indicate how serious the
problem is for you by checking the box on the five-point scale "a slight problem" to "a very serious
problem". If not, then check the box "not applicable".
QSC - R10
1.I feel tired and weak.
2. I am in pain.
3. I feel physically imperfect.
4. I have too few opportunities to speak with a professional
about my psychological distress.
5. I am afraid that my disease will spread/recur.
6. It is difficult for my partner to empathize with my situation.
7. My sleep is disturbed.
8. It is harder for me to take part in recreation activities
(e.g. sports) now than it was before I became ill.
9. I do not feel well informed about my disease/treatment.
10. I feel tense and/or nervous.

Table 1 .Questionnaire on Stress in Cancer Patients - short form (QSC-R10, according to Book et al., 2011 [4]).

All statistical analyses were conducted with SPSS, version 19 (SPSS, Inc., Chicago, IL, USA). For the sample characteristics and items of the QSC-R10, descriptive statistics were conducted. Fisher's exact test was used to verify the differences in need for psycho- oncological support between 1 st and 2 nd survey. For all quantitative analyses, $\mathrm{p}<0.05$ was considered to indicate statisticalsignificance.

\section{Results}

\section{Baseline patient characteristics}

The median age in the entire study group was 59 years (range: 30-82 years). Patients intended for curative treatment $(\mathrm{N}=61)$ had a median age of 55.0 years (range: 35-79 years) and were significantly younger ( $p=$ $0,013)$ than those subjected to palliative treatment $(\mathrm{N}=33)$ with a median age of 66.0 years (range: $30-82$ years).

The vast majority of patients $(\mathrm{n}=59 ; 98 \%)$ with curative treatment intention exhibited a Karnofsky Performance Status (KPS) between 80 and 100 percent, meaning that they were able to work and carry on normal activities. Among patients receiving palliative treatment, 29 (88\%) exhibited a high KPS of $80-100$ percent $(p=0,052)$.
Consistent to the younger age of patients with curative treatment intention, a significantly higher proportion out of this group were still working $(\mathrm{n}=32 ; 53 \%)$ compared to those in the palliative setting $(\mathrm{n}=6 ; 18 \%)$ $(\mathrm{p}=0,000)$. With 25 out of 32 individuals $(78 \%)$, the majority of patients with recurrent/metastatic disease were retired, versus 19 out of 61 the patients $(31 \%)$ in the curative cohort.

In general, the majority of the analyzed patients were living together with a partner and/or with their children -instead of living alone. This number was similar among patients with curative treatment intention $(n=48 ; 79 \%)$ compared to patients receiving palliative therapy $(n=27 ; 82 \%)(p=0,718)$.

Psycho-oncological burden in breast cancer patients

Among all breast cancer patients included, a total number of 39 (41,5\%) suffered from psycho-oncological distress at the time of the first survey (Figure 1). According to this, no signs of stress were reflected in the initial evaluation of the QSC-R10 in 55 patients $(58,5 \%)$. There was no difference in median age between burdened and not-burdened patients $(\mathrm{p}=0,172)$.

The average lead time time between the first and the second survey was 40,6 weeks (median:

30 weeks, range: $2-138$ weeks). During the second survey, $26(66,6 \%)$ of the initially burdened patients were still identified to require psycho-oncological support (Figure 1). Thirteen (33,3\%) patients no longer showed symptoms of psycho-oncological impairment. Among the $55(58,5 \%)$ initially burden-free patients, $41(74,5 \%)$ still showed no hints of psycho-oncological distress at the time of the second survey. However, fourteen individuals out of this group $(25,5 \%)$ exhibited significant signs of psycho-oncological impairment according to the results of the second survey (Figure1).

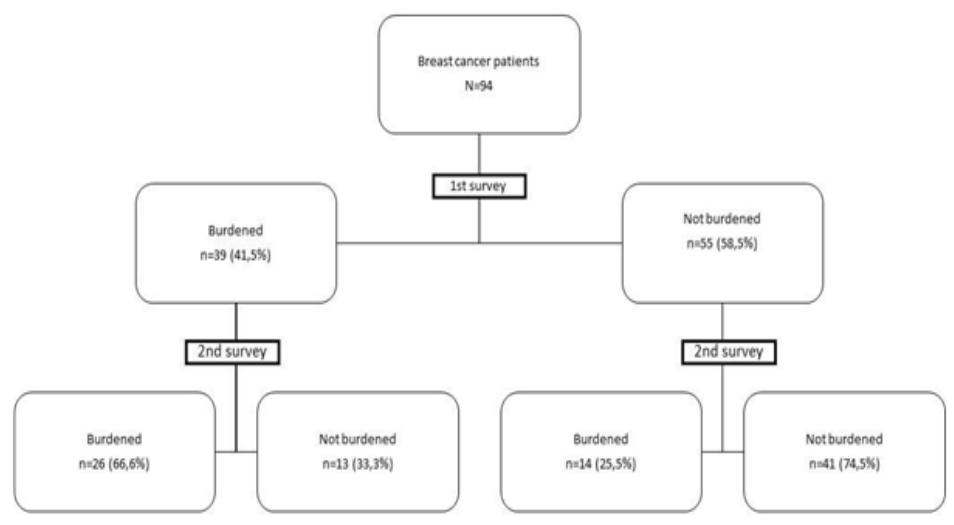

Figure 1 .Psycho-oncological burden in breast cancer patients throughout the observation period.

Psycho-oncological burden by treatment intention

According to the evaluation of the QSC-R10 by treatment intention, no significant differences were observed in the proportion of patients with or without signs of psycho-oncological burden at the time of the first survey. As shown in Figure 3, 26 out of 61 patients in the curative setting $(42,6 \%)$ and 13 out of 33 patients intended for palliative therapy $(39,4 \%)$ were burdened $(\mathrm{p}=0.828)$. Although a significant improvement was observed in the curative setting $(p=0.001)$ the majority of initially burdened patients still required psycho-oncological support at the time of the second survey $(15 / 26)$. In total, fifteen of 61 patients in the curative and eleven of 33 patients in the palliative cohort exhibited symptoms of psycho-oncological distress when completing the second QSC-R10 questionnaire. This means that in two thirds of all initially burdened patients (i. e. $26 / 39 ; 67 \%$ ) the psycho-oncological distress was not resolved after a median lead time of 30 weeks (range: $2-138$ weeks): $15 / 26$ patients $(57,7 \%)$ in the curative and $11 / 13$ patients $(84,6 \%)$ in the palliative setting (Figure 2 ). Moreover, 
fourteen of 55 patients $(25,4 \%)$, who initially showed no signs of psycho-oncological burden, were in need of support at the time of the second survey: five out of 35 patients $(14,3 \%)$ who were receiving curative therapy and nine out of twenty patients $(45,0 \%)$ who were subjected to palliative treatment, resulting in a significant worsening in this subgroup during the observed period of time $(p=0.032)$.

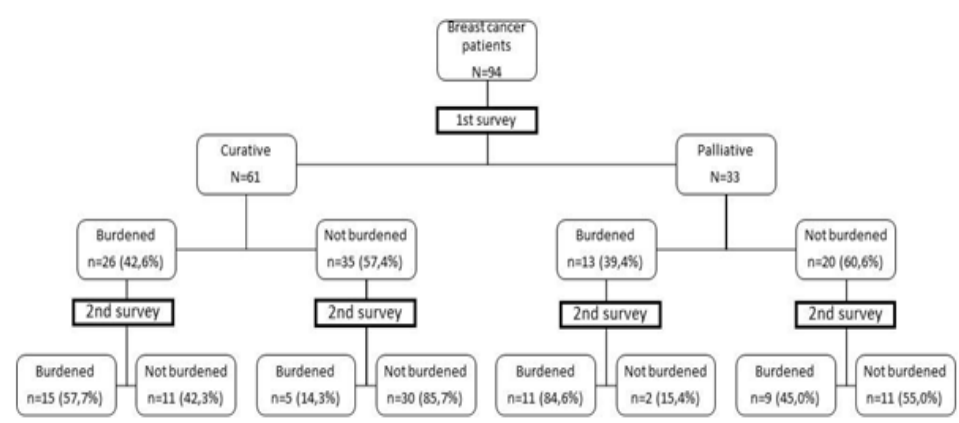

Figure 2 .Psycho-oncological burden in breast cancer patients by treatment intention

\section{Discussion}

The present prospective questionnaire-based study is one of the first that monitored breast cancer patients in the long-term regarding their need for psycho-oncological support. In addition to the main objectives of medical cancer treatment, psycho-oncological aspects have recently come more and more into the clinical focus [8]. Many patients successfully repress their disease throughout the intensive primary cancer treatment. However, following the completion of this treatment phase, patients may feel particularly vulnerable and face threats to their identity [9]. To its full extend, psycho-oncological burden often occurs for the first time when patients are no longer in contact with their physician as frequently as throughout the intensive primary treatment. As shown in the present study, psycho-oncological burden was already observed in $>40 \%$ of the breast cancer patients at an early stage during the course of the disease. Diagnosis at this stage can diminish the risk of potential escalation of distress in the future [10]. To ensure adequate support in all phases of the cancer experience, psycho- oncological assessment is thus required from the time of diagnosis and in the long-term following the completion of the primarytreatment.

It is important that psycho-oncological burden in cancer patients is diagnosed, as distress and psychiatric morbidity are known to be associated with poorer outcomes including mortality [6]. In this context, Chan et al. (2014) observed a survival benefit of 2.24 months, or 67 days in cancer patients without psychiatric morbidity [6]. Various studies have shown a direct correlation between the course of treatment and the patients' quality of life $(\mathrm{QoL})[11,12,13]$. QoL is an important measure of treatment success because the treatment can affect the patient's everyday life and can cause serious harm, which can outweigh the advantages it is supposed to achieve [14]. Therefore, the awareness among clinicians concerning the relevance of maintaining and improving the QoL of patients undergoing cancer treatment is increasing [15].

In consideration of the limited financial and human resources in the health care system, it is necessary to use adequate instruments for the identification of patients in need of psycho- oncological support [8]. In this context, the QSC-R10 has shown good results specifically in cancer patients [4]. This short form of the standard version QSC-R23 was developed

especially for the purpose of distress screening. It can be completed by cancer patients themselves within a few minutes. An electronic version of the questionnaire, the QSC-R10e, is also available. It is answered online on a computer and the result can be seen immediately [16]. Due to its brevity and the minimal effort for answering and evaluating the results of the questionnaire, the QSC-R10 is well suitable for repeated and long-term use in cancer patients. Generally, various types of therapy have proven to be effective in the patients' psychological condition. Alternative non-verbal forms of therapy are increasingly used because cancer disease is often accompanied by demanding experiences that are not always easily captured in words [12]. In this context, Svensk et al. (2009) demonstrated in a randomized controlled trial that art therapy improved the experienced QoL among women undergoing treatment for breast cancer[12].

Finally, the choice of therapy used to offer psychological support seems to be less significant. In contrast, it is of primary importance to diagnose burden and to subsequently create possibilities for the affected patients to express their emotions, and in particular to process their concerns and fears with perspectives for the future [12]. Even if the patient is cared by family and friends, professional external support is necessary because the affected ones are often worried that family and friends will be too emotionally involved in their concerns and fears [17]. All in all, continued assessment of the patients' needs and symptoms provide the basis for purposeful counselling [18]. In summary, the fundamental importance of the long- term monitoring should be emphasized once more because, as shown by the results of the present study, psycho-oncological burden is present in a large proportion of the affected patients' even long time after the primary diagnosis. In this context, it seems to be of particular interest that psychological distress was recorded during the second survey in approximately one quarter of all patients who initially did not show any signs of psychological impairment. This argues in favor that psycho-oncological burden may well occur in a small but significant subset of initially unaffected breast cancer patients after a prolonged period of time. Thus, psycho-oncological support should be perpetuated in order to prevent patients at risk to develop a late-onset form of psycho-oncological distress from experiencing this clinically demanding and QoL-deterioratingcondition.

Even though the present study is one of the very first that monitored breast cancer patients over a prolonged period of time with regard to their need of psycho-oncological support in an outpatient setting, it clearly owns limitations such as the small sample size and the fact that this research was conducted in two oncological practices only. Nonetheless, to all our best knowledge our longitudinal study represents the first of its kind performed in a real-world population of breast cancer patients treated for both primary and metastatic disease thus underlining its potential value for the clinical routine. Moreover, our results indicate the importance of long-term monitoring the psycho-oncological burden of cancer patients and give reason for further, comparable studies investigating an enlarged population of patients for an equal or even longer observationtime.

In summary, psycho-oncological burden is very common in breast cancer patients, independent of whether the patients are treated with a curative or palliative intention. The need for psycho-oncological support not only exists at the time of the first confrontation with the disease, but also after the completion of the intensive primary cancer treatment. In the future, this temporal dimension has to be addressed more sufficiently, in order to be able to support patients in particular after the completion of the irintensivetherapy.Thus, the long-term monitoring of cancer patients with the QSC-R10 is strongly recommended, because the questionnaire adequately reflects the psycho-oncological condition of the affected patients, and it requires only minimal effort to be answered Furthermore, the QSC-R10 is very well accepted among healthcare professionals andpatients.

\section{Conclusion}

The psychooncological burden is common in breast cancer patients and is influenced by various factors, including treatment intention. The QSC-R10 has proven to be an appropriate instrument to identify these factors, and to reveal the need for psychooncological support. Timely initiation of adequate measures as well as long-term monitoring beyond the completion of the intensive primary cancer treatment are necessary to meet the individual need of breast cancerpatients. 
Funding This study was funded by ZytoService, Hamburg, Germany. Previous publications Parts of this study were presented at the ASCO Annual Meeting (abstract \# e20565), Chicago, IL, USA, May 29-June 2, 2015, and at the MASCC/ISOO Annual Meeting, Copenhagen, Denmark, June 25-27, 2015 (abstract \# 18-51-P).

Author contributions All listed authors have seen and approved the final version of the manuscript. All authors have met all three of the following criteria for authorship, thereby accepting public responsibility for appropriate portions of the content:

1.substantial contributions to conception and design, or acquisition of data, or analysis and interpretation ofdata;

2.drafting the article or revising in critically for important intellectualcontent;

3.approval of the version to be published and all subsequentversions.

\section{References}

1. Robert Koch Institut (RKI). Krebs in Deutschland 2009/2010,2013 Accessed 05 May 2015.

2. Yoo GJ, Levine EG, Aviv $\mathrm{C}$ et al. Older women, breast cancer, and social support. Support Care Cancer 2010; 18: 1521-1530 .

3. Söllner W, Maislinger S, König A et al. Providing psychooncological support for breast cancer patients based on screening for distress within a consultation-liaison service. Psychooncology 2004; 13: 893-897 .

4. Book K, Marten-Mittag B, Henrich G et al. Distress screening in oncology - evaluation of the Questionnaire on Distress in Cancer Patients - short form (QSC-R10) in a German sample. Psychooncology 2011; 20 (3): 287- 293. DOI:10.1002/pon.1821.

5. Herschbach P et al., Hrsg. Screeningverfahren in der Psychoonkologie. Testinstrument zur Identifikation betreuungsbedürftiger Krebspatienten. Eine Empfehlung der PSO für die psychoonkologische Behandlungspraxis. Berlin: Deutsche Krebsgesellschaft e.V., 2010 .

6 Chan CM-H, Ahmad WAW, Yusof MM et al. Distress and psychiatric morbidity in cancer patients: prevalence and association with mortality in a two-year longitudinal study. ASCO Annual Meeting 2014; Abstract No:9529.

7 Arbeitsgemeinschaft der Wissenschaftlichen Medizinischen Fachgesellschaften e.v (AWMF) Interdisziplinäre S3-Leitlinie für die Diagnostik, Therapie und Nachsorge des Mammakarzinoms. Langversion 3.0, Aktualisierung 2012. 2012; AWMF-Register- Nummer:032$045 \mathrm{OL} ; A$ Accessed 06 May2015.
8. Zenger M, Ernst J, Götze H etal. The need for psychooncological support and the desire for counselling in prostate cancer patients $-\mathrm{a}$ longitudinal study. Z Med Psychol 2010; 19: 51-59

9. Foster C, Fenlon D. Recovery and self-management support following primary cancer treatment. Br J Cancer 2011; 105: 21-28 .

10. Kovac A, Petrović SP, Nedeljković $M$ et al. Post-operative condition of breast cancer patients from standpoint of psycho-oncology preliminary results. Med Pregl 2014; 67 (1-2): 43-48 .

11. Okamura M, Yamawaki S, Akechi $\mathrm{T}$ et al. Psychiatric Disorders Following First Breast Cancer Recurrence: Prevalence, Associated Factors and Relationship to Quality of Life. Jpn J Clin Oncol 2005; 35 (6): 302-309.

12. Svensk A-C, Öster I, Thyme KE et al. Art therapy improves experiences quality of life among women undergoing treatment for breast cancer: a randomized controlled study. Eur J Cancer Care 2009; 18: 6977. DOI:10.1111/j.1365-2354.2008.00952 .

13. Akechi T, Okuyama T, Endo C et al. Patient's perceived need and psychooncological distress and/or quality of life in ambulatory breast cancer patients in Japan. Psychooncology 2011; 20 (5): 497-505 .

14. Shimozuma K. Quality of life assessment. Breast cancer 2002; 9: 100-106 .

15.Hakamies-Blomqvist L, Louma ML, Sjöström J et al. Timing of quality of life (QoL) assessments as a source of error in oncological trials. J Adv Nurs 2001; 35: 709-716.

16. Herschbach P. Distress screening with the questionnaire on stress in cancerpatients.Onkologie 2008; 31 (suppl. 1): 1- 211

17. Serlin IA, Classen C, Frances B, Angell K. Symposium: support groups for women with breast cancer: traditional and alternative expressive approaches. The Arts in Psychotherapy 2000; 27: 123-138 .

18. Schmid-Büchi S, Halfens RJG, Dassen $T$ et val. Psychosocial problems and needs of posttreatment patients with breast cancer and their relatives. Eur J Oncol Nurs 2010; 15 (3): 260-266 .

19. Herschbach, P., Weis, J. (Hrsg.) (2010). Screeningverfahren in der Psychoonkologie Testinstrumente zur Identifikation betreuungsbedürftiger Krebspatienten. Eine Empfehlung der PSO für die psychoonkologische Behandlungspraxis, Berlin 2010, 2. Auflage . 